\title{
From Hipparcos to Gaia
}

\author{
L. Eyer ${ }^{1}$, P. Dubath ${ }^{1,2}$, S. Saesen ${ }^{1}$, D.W. Evans ${ }^{3}$, L. Wyrzykowski ${ }^{3}$, \\ S. Hodgkin ${ }^{3}$ and N. Mowlavi ${ }^{1,2}$ \\ ${ }^{1}$ Geneva Observatory, CH-1290 Sauverny, Switzerland \\ email: Laurent.Eyer@unige.ch \\ ${ }^{2}$ ISDC, Department of Astronomy, University of Geneva, CH-1290 Versoix, Switzerland \\ ${ }^{3}$ Institute of Astronomy, Cambridge University, Cambridge, UK \\ Invited Talk
}

\begin{abstract}
The measurement of the positions, distances, motions and luminosities of stars represents the foundations of modern astronomical knowledge. Launched at the end of the eighties, the ESA Hipparcos satellite was the first space mission dedicated to such measurements. Hipparcos improved position accuracies by a factor of 100 compared to typical ground-based results and provided astrometric and photometric multi-epoch observations of 118,000 stars over the entire sky. The impact of Hipparcos on astrophysics has been extremely valuable and diverse. Building on this important European success, the ESA GAIA cornerstone mission promises an even more impressive advance. Compared to Hipparcos, it will bring a gain of a factor 50 to 100 in position accuracy and of a factor of 10,000 in star number, collecting photometric, spectrophotometric and spectroscopic data for one billion celestial objects. During its 5-year flight, GAIA will measure objects repeatedly, up to a few hundred times, providing an unprecedented database to study the variability of all types of celestial objects. GAIA will bring outstanding contributions, directly or indirectly, to most fields of research in astrophysics, such as the study of our Galaxy and of its stellar constituents, and the search for planets outside the solar system.
\end{abstract}

Keywords. space vehicles, surveys, stars: variables: other, astrometry, techniques: photometric, techniques: spectroscopic

\section{Introduction}

Time domain plays a central role in the Hipparcos and GaIA missions. Indeed, to determine parallaxes and proper motions of stars, repeated observations of positions are required. The accuracy of ground-based astrometric measurements are limited because of atmospheric distortions, telescope deformations and difficulties related to the definition of the reference frame. Even if a set of distant stars is used, they may have streaming motions or residual parallaxes, leading to inaccuracies in parallax measurements.

In the realms of space, deformations caused by the presence of the atmosphere or gravity are avoided altogether. The issue of the reference frame is solved in a similar manner for both HipPARCOS and GAIA. The basic measurements are wide angles between celestial targets. As the spacecraft is scanning the sky over an extended period of time, many such angles with many orientations are typically measured for a given target. The whole sphere is then reconstructed using some mathematical formulations, solving for the astrometric parameters of all targets globally. In that way, a full-sky positional frame of unprecedented accuracy can be self-calibrated (Lindegren \& Bastian 2011).

Both Hipparcos and GaIA are projects of the European Space Agency (ESA). Other astrometric missions have been proposed, notably the USA's FAME and SIM, but were not in the end pursued. There is, however, a Japanese astrometric project, JASMINE (see http://www.jasmine-galaxy.org/index.html), the first step of which 
is a nano-satellite to be launched in 2013. JASMINE should achieve HIPPARCOS-quality accuracies. Although the principles of astrometric measurements are similar for both HipPARCOS and GAIA, their scientific cases are different; the science case for HipPARCOS was mostly stellar, whereas that for GAIA is more oriented towards the Galaxy, aiming to study its structure and formation history. However, GAIA will also have a very significant impact on asteroid studies, stellar astronomy and fundamental physics.

Both missions also provide very valuable data sets for variability studies. The cadence of the observations is imposed by optimizing the satellite's scanning routines for best astrometric performance. It turns out, however, that the resulting time-sampling is rather favourable for period determination (Eyer \& Mignard 2005), since the one-day and one-year aliases (and related ones) which tend to plague ground-based observations are absent, and other aliases relating to spacecraft characteristic periods are of rather low amplitude. In addition, both missions are performing a homogeneous whole-sky survey with a single set of instruments and with fully standard data reduction procedures, thereby producing a very coherent data set. Though the results of HIPPARCOS were published in 1997, they are still unsurpassed in extent and quality. That is true, not only for astrometry but also for variability studies: HiPPARCOS is still the only whole-sky survey with a published systematic variability analysis.

\section{HipPARCOS}

The Hipparcos mission collected observations for 118,000 stars down to magnitude $H_{p}=12.4$, grouped into two main samples:

(a) A whole-sky survey sample of 52,000 stars brighter than $7.9 \mathrm{~V}+1.1 \sin (b)$ magnitude for blue stars with $B-V<0.8$ and brighter than $7.3 V+1.1 \sin (b)$ for redder stars with $B-V \geqslant 0.8$ ( $b$ is the Galactic latitude).

(b) A set of 66,000 objects selected on the basis on their scientific interest.

The properties of these objects (position, proper motion, magnitude, colour, spectral and variability types, radial velocities and multiplicity) were compiled and published in the Hipparcos Input Catalogue (ESA 1992). There were also observational campaigns to collect complementary information during the mission. For example, the AAVSO (American Association of Variable Star Observers) measured the brightness of long-period variables in order to determine the correct exposure times for HIPPARCOs for those brighter than $H_{p}=12.4$. In another campaign the $H_{p}$ band was re-calibrated by measuring $\mathrm{R}, \mathrm{N}$ and $\mathrm{C}$ stars simultaneously from the ground and from space.

The satellite was launched from Kourou (French Guiana) on an Ariane-4 rocket in 1989. The length of the mission was 3.3 years, during which an average of 110 measurements per object were collected. The final results were published in a 17 -volume catalogue in 1997 (ESA 1997). The mission realized its original goal of obtaining astrometry at the milli-arcsecond level. In addition, the Hipparcos Star Mapper provided measurements which led to the Tycho (ESA 1997) and Tycho-2 (Høg et al. 2000) catalogues, which contain astrometric and photometric $\left(B_{T}\right.$ and $\left.V_{T}\right)$ measurements for 1 and 2.5 million stars, respectively. As expected, the Tycho epoch photometry in $B_{T}$ and $V_{T}$ has been more difficult to handle than the $H_{p}$-band photometry from the main mission, and has led to a relatively low number of variable stars (Piquard et al. 2001). Ten years after the publication of the Hipparcos Catalogue a new reduction of the astrometric data was undertaken (van Leeuwen 2007). Various systematic effects were reduced for bright stars, leading to errors more consistent with the photon noise limits. The project scientist of Hipparcos, Michael Perryman, wrote two books about the mission (Perryman 2009 and 
Perryman 2010). The first summarizes and highlights the scientific harvest of HiPPARCOS during the 10 years following the publication of the catalogue. The second narrates the fantastic human, technological and scientific adventures of the HipPARCOs mission.

\subsection{A Note on the Pleiades Distance Derived from Hipparcos}

Distances of open clusters have been based upon HipPARCos parallaxes. For the Pleiades, the distance of $118.3 \pm 3.5$ pc thus derived (van Leeuwen 1999) was in contradiction to the value of $132 \pm 4 \mathrm{pc}$ obtained by the customary main-sequence-fitting technique (Meynet et al. 1993). Other independent determinations (Pan et al. 2004; Zwahlen et al. 2004; Munari et al. 2004; Soderblom et al. 2005) favour the greater distance. Although, at the level of individual stars, the distances determined via the HiPPARCOs parallax measurements are acceptable within the individual error estimates, the discrepancy becomes more acute when averaging. The new HipparCos reduction by van Leeuwen (2007) confirmed the smaller value of $120 \pm 1.9 \mathrm{pc}$ (van Leeuwen 2009). This is the kind of debate that GAIA will close. Unfortunately, for some individual stars such as Atlas, GAIA will not be able to provide a distance determination because its survey is limited to $V \sim 6$ mag.

\section{GAIA}

The GAIA programme anticipates astrometric, photometric, spectrophotometric and spectroscopic measurements for one billion celestial objects with magnitudes between $V \sim 6-20$. The mission is designed to last for 5 years, with a possible 1-year extension; the launch is currently planned for 2013. The number of measurements accumulated after 5 years varies between 40 and 250 per object, depending mostly on ecliptic latitude, and expects to average 70 (the estimate takes into account the dead-time estimation). Spectroscopic measurements with a resolution of 11,500 will be obtained for stars brighter than 16-17 mag. Recent performance estimates can be found on the GAIA Webpaget. It is important to note that the astrometric accuracies quoted were derived as errors in the parallax at the end of the mission.

It was realized early in the preparation of the mission that processing all the data will be a challenge of the highest order. People interested in certain aspects created Working Groups, which later evolved into Coordination Units (Mignard et al. 2008). There are currently 8 Coordination Units (CU); one of them, CU7, is responsible for all aspects relating to variability analyses of the data. The CU7 analyses start with calibrated data provided by other Coordination Units, but the variability and related time-series analyses have impacts on most Coordination Units. One additional Coordination Unit is currently being formed to develop the catalogue access interface for the scientific community. The GAIA Data Processing and Analysis Consortium (DPAC) now includes more than 500 scientists and software engineers. Our estimates of the number of variable sources that GAIA will detect range from 50 million to 150 million. The rather large range reveals limitations of our knowledge in the domain of variable phenomena, but it also reflects uncertainties in the ultimate precision reached once the satellite is in space. Some effort is currently being spent to improve these estimates using results from other space missions such as KEPLER and CoRoT. The diversity of variable phenomena is illustrated by the variability tree presented by Eyer \& Mowlavi (2008). GAIA will detect most variability types from that tree.

$\dagger$ http://sci.esa.int/science-e/www/area/index.cfm?fareaid=26 


\subsection{Variability Processing and Analysis}

CU7 is in charge of analyzing all variable objects that appear above a certain variability threshold. Specific criteria are set for detecting particular phenomena such as short-period variables, solar-like magnetic variables, planetary transits and periodic small-amplitude variables. Those characteristics are taken into account to lower the variability thresholds set for general purposes. That special variability detection can therefore select variable stars that would be considered constant based on the general variability criteria.

Once an object is considered a candidate for variability, its behaviour is characterized by certain parameters. Simple models such as a trend or periodicity model are tested to see if they can explain the variability. This characterization task produces a set of parameters ("attributes") that are used in the next step of the processing, which is the classification. The classification algorithms compute membership probabilities for each object on the basis of the specific values of the attributes. The intention is to use supervised, unsupervised and semi-supervised methods. Different supervised methods have been evaluated using Hipparcos data, and "random forest" proves to be one of the best algorithms in terms of overall performance (Dubath et al. 2011).

Once an object is classified, then if it belongs to a certain specific group of variables, further specific analysis is carried out. The different classes that will thus be treated in more detail currently include eclipsing binaries, solar-like variability (powered by magnetic fields), rotation-induced variable stars, RR Lyrae/Cepheid stars, long-period variables, main-sequence pulsators, compact oscillators, pre-main sequence oscillators, microlensing, cataclysmic variables, rapid phases of stellar evolution, and active galactic nuclei. In that step, some attributes will be refined and additional parameters computed, but another important activity is to evaluate the quality of the classification, since contamination and completeness are two fundamental criteria.

\subsection{Science Alerts}

DPAC is preparing a system for science alerts (Wyrzykowski \& Hodgkin 2011). Science alerts are defined as events where "the science data would have little or no value without quick ground-based follow-up". Different types are foreseen from different instruments: astrometric alerts (including fast moving objects), photometric, and spectroscopic alerts. Examples of objects that could constitute photometric alerts include supernovæ $(6,000$, with 2,000 before they peak), microlensing events (1,000, with some having an astrometric signature), cataclysmic variables (novæ, dwarf novæ), eruptive stars (Be, $\mathrm{RCrB}$ stars, FU Ori) and possibly gamma-ray bursts, including orphan afterglows. The detection of these photometric alerts will be performed in near-real-time GAIA broad-filter photometry. The classification, however, will utilize all other data available immediately from GAIA, including preliminarily calibrated low-resolution spectra. This will assure the best possible recognition of an alert. For supernovæ, for example, it can provide a preliminary type and an estimate of the red-shift. GAIA will also provide alerts of Near Earth Objects and potentially hazardous asteroids, owing to its superb astrometry capability. One of the huge challenges for these science alerts is how to maximize completeness and avoid contamination from other uninteresting objects.

Since the data might sometimes accumulate on board the satellite before being transmitted to Earth, there are time constraints which limit in practice the window within which alerts can be treated. This time window is between a few hours and two days. The alerts will not start from the first day of operation, but only after a 3-month verification phase. The goal is to verify the quality of the products of the alert pipeline and-more precisely - the probabilities associated with the classification of these alerts. A group of ground-based telescopes is being set up to assist with the verification phase. Furthermore, 
once the data are on public release it is agreed that scientific follow-up has to be organized in advance. Such structuring of the ground-based effort is being done now. With the development of large ground-based surveys, the specificities of the GAIA alert system should be compared to others such as PTF, CRTS, SKyMAPPER and Pan-STARRS, and tuned to complement them as well as possible.

There has been a proposal to introduce a "Watch List", that is, a list of targets suggested by interested scientists. If one of those targets behaves in an unexpected manner, an alert will be sent to the scientist(s) in question so that a follow-up can be organized. That requires good coordination among groups with different scientific interests, but is an effective way of enhancing the scientific return of GAIA for selected objects.

\section{Conclusions}

The Hipparcos mission has been a tremendous success. If the results of the GAIA mission reach current expectations, there is no doubt at all that its multi-epoch survey of astrometry, photometry, spectrophotometry and spectroscopy will have considerable impacts on astrophysics. But we should recognize that GAIA is not the only large-scale survey which is collecting data now, or is planned to do so: Pan-STARRS, LSST, OGLE, Sky-Mapper, PTF and CRTS are not all. GAIA will nevertheless make a significant and unique contribution because no other survey will reach the astrometric accuracy of GAIA. In return GAIA will benefit from the other surveys, especially those having photometric systems that extend into the ultraviolet or infrared.

A large public spectroscopic survey relating to GAIA was selected by ESO for the VLT; it starts in 2012 January and is expected to have 30 nights per semester for four years, with an additional fifth year subject to progress review.

\section{Acknowledgements}

We thank Michel Grenon, Joshua Bloom and Pierre North for valuable discussions.

\section{References}

Dubath, P., et al. 2011, MNRAS, 414, 2602

ESA. 1992, The Hipparcos Input Catalogue ESA SP-1136

ESA. 1997, The Hipparcos and Tycho Catalogues, ESA SP-1200

Eyer, L. \& Mignard, F. 2005, MNRAS, 361, 1136

Eyer, L. \& Mowlavi, N. 2008, J. Phys. Conf Series, 118, 012010

$\mathrm{H} \varnothing \mathrm{g}$, E., et al. 2000, A\&AA, 355, L27

Lindegren, L. \& Bastian, U. 2011, EAS Publications Series, 45, 109

Meynet, G., Mermilliod, J.-C., \& Maeder, A. 1993, A\&AS, 98, 477

Mignard, F., et al. 2008, IAU Symposium, 248, 224

Munari, U., et al. 2004, A\&BA, 418, L31

Pan, X., Shao, M., \& Kulkarni, S. R. 2004, Nature, 427, 326

Perryman, M. 2009, Astronomical Applications of Astrometry: Ten Years of Exploitation of the Hipparcos Satellite Data (Cambridge, UK: Cambridge University Press)

Perryman, M. 2010, The Making of History's Greatest Star Map (Berlin: Springer-Verlag)

Piquard, S., et al. 2001, A\&SA, 373, 576

Soderblom, D. R., et al. 2005, AJ, 129, 1616

van Leeuwen, F. 1999, A\&SA, 341, L71

van Leeuwen, F. 2007, ASSL (Dordrecht: Springer), Vol. 350

van Leeuwen, F. 2009, $A \mathscr{E} A$, 497, 209

Wyrzykowski, L. \& Hodgkin, S. 2011, arXiv:1112.0187

Zwahlen, N., 2004, A\&A, 425, L45 